\title{
Local Investigation of Femtosecond Laser Induced Dynamics of Water Nanoclusters on Cu(111)
}

\author{
Michael Mehlhorn, ${ }^{1}$ Javier Carrasco, ${ }^{2,3}$ Angelos Michaelides, ${ }^{2}$ and Karina Morgenstern ${ }^{1}$ \\ ${ }^{1}$ Institut für Festkörperphysik, Leibniz Universität Hannover, Appelstraße 2, 30167 Hannover, Germany \\ ${ }^{2}$ London Centre for Nanotechnology and Department of Chemistry, University College London, London WC1E 6BT, United Kingdom \\ ${ }^{3}$ Fritz-Haber-Institut der Max-Planck-Gesellschaft, Faradayweg 4-6, 14195 Berlin, Germany
}

(Received 17 February 2009; published 10 July 2009)

\begin{abstract}
We explore the dynamics of low temperature interfacial water nanoclusters on $\mathrm{Cu}(111)$ by femtosecond-laser excitation, scanning tunneling microscopy and density functional theory. Laser illumination is used to induce single molecules to diffuse within water clusters and across the surface, breaking and reforming hydrogen bonds. A linear diffusion probability with laser fluence is observed up to $0.6 \mathrm{~J} / \mathrm{m}^{2}$ and we suggest that diffusion is initiated by hot electron attachment and detachment processes. The density functional calculations shed light on the detailed molecular mechanism for water diffusion that is determined by the local structure of the water clusters.
\end{abstract}

PACS numbers: 68.37.Ef, 68.43.Bc, 82.30.Rs, 82.53.St

There is broad interest in supported water-ice and the mechanims of energy and charge transport through it in areas as diverse as the environmental sciences and astrochemistry. For example, ionizing radiation can impact upon the chemical composition of Earth's upper atmosphere (e.g. its ozone density) by triggering chemical processes at the surfaces of cold ice covered grain particles. In the laboratory this situation can be mimicked by preparing ultrathin water-ice overlayers or clusters on a metal support under ultrahigh vacuum (UHV) conditions and probing them by driving conduction electrons from the support into the water overlayer with a laser. Given the enormous body of knowledge on the structure and properties of such metal supported water overlayers, they can provide well-defined model systems. However, so far only one study has been aimed at exploring the impact of femtosecond (fs) laser irradiation on nuclear motion within water overlayers [1]. Specifically, at high laser fluence (tens of $\mathrm{J} / \mathrm{m}^{2}$ ) Backus et al. forced water molecules to desorb from an amorphous water overlayer on a Pt surface, detecting the (ensemble of) desorbed molecules with mass spectrometry. The molecular-scale details of such processes, in terms of the dynamics of individual water molecules and clusters, are, however, still unexplored. Moreover, it is not known how water overlayers will behave at much lower laser fluences when the molecules are not expected to desorb from the surface.

Through the combination of a femtosecond laser with a scanning tunneling microscope (STM) [2,3] it is now possible to resolve details of the dynamics induced by fs lasers at surfaces on specific sites, i.e., on the length scale of molecules, rather than measuring the average response of an ensemble of molecules at different surface sites [4]. This technique affords the opportunity to follow the changes induced to individual molecules by laser excited electrons in their specific local environment and the future possibility of laser-controlled dynamics on a single mole- cule level. However, so far, it has only been applied in two qualitative studies (CO diffusion [2] and chloronitrobenzene diffusion and rotation [3]) and has not been used to make quantitative measurements, such as a determination of diffusion or reaction probabilities.

Here, we apply the fs laser-STM combination to explore water nanoclusters. Specifically we examine a set of wellcharacterized nanoclusters that form upon exposure of water to $\mathrm{Cu}(111)$ at low temperatures under UHV [5]. We investigate fs-laser induced changes at much lower fluence than explored in [1] and find that even at low fluences $\left(<1 \mathrm{~J} / \mathrm{m}^{2}\right)$ water diffusion can be induced and hydrogen bonds broken and reformed. The dominant process observed is the diffusion of individual water monomers within the water nanoclusters, a suggestion that is supported by density functional theory (DFT) calculations. This study gives insight into the hydrogen-bond rearrangement behavior of individual molecules upon low intensity radiation at cryogenic temperatures, where thermal dynamics is frozen.

The measurements are performed with a custom-built system [3] that combines the single molecule spatial resolution of a low temperature STM in UHV with the ultrafast surface dynamics driven by fs-laser excitation. The base temperature of the STM during laser operation is below $7 \mathrm{~K}$. The femtosecond-laser system delivers $30 \mathrm{fs}$ (FWHM) pulses at $796 \mathrm{~nm}$ (band width $40 \mathrm{~nm}$ ) with a pulse energy of up to slightly more than $30 \mathrm{~nJ}$ at $10.67 \mathrm{MHz}$ repetition rate. The pulses are frequency doubled to provide higher excitation energy and photon absorption in $\mathrm{Cu}(111)$. The Gaussian profile of the elliptical laser spot is $10 \mu \mathrm{m} \times$ $30 \mu \mathrm{m}$ (FWHM) at the sample, which allows to achieve an absorbed fluence of some $\mathrm{J} / \mathrm{m}^{2}$. In a parallel setup we determine $\tau_{400 \mathrm{~nm}} \approx(40 \pm 5)$ fs. The single crystalline $\mathrm{Cu}(111)$ surface is cleaned by cycles of $\mathrm{Ne}^{+}$sputtering and annealing. Ultrapure $\mathrm{D}_{2} \mathrm{O}$ is further cleaned in vacuum by freeze-pump-thaw cycles. A few percent of a $\mathrm{D}_{2} \mathrm{O}$ bilayer (BL) is deposited from the vapor pressure through 
a molecular tube doser at a rate of $0.16 \mathrm{BL} / \mathrm{min}$ to the surface held at $17 \mathrm{~K}$.

At low coverages water adsorbs as monomers and small clusters on $\mathrm{Cu}(111)$ [and $\mathrm{Ag}(111)$ ]. The most common adsorbed structures are clusters with six to nine water molecules [5]. The basic building block of these structures is a buckled cyclic water hexagon [6] which becomes "hydrated" by one, two, or three water molecules thus leading to heptamers, octamers, and nonamers [5]. Figure 1 shows STM images, in which monomers, a hexamer, octamers, and nonamers can be identified along with illustrations of the structures of the various clusters. The STM images of these clusters are dominated by the additional peripheral molecules, each of them imaged as a protrusion. At these temperatures and up to $40 \mathrm{~K}$ the clusters are thermally stable on the time scale of the measurement as determined by in situ annealing up to $40 \mathrm{~K}$.

Having set the stage, such a water-Cu sample was illuminated with the fs laser at low fluences $\left(<1 \mathrm{~J} / \mathrm{m}^{2}\right)$. Figure 2 shows STM images of the surface throughout a series of consecutive fs-laser illuminations. The two top panels, with a step in the upper left-hand side, illustrate that exactly the same part of the surface is imaged before and after illumination even though the STM tip is retracted (200 $\mathrm{nm}$ vertically and $1 \mu \mathrm{m}$ laterally) during laser illumination so as to avoid tip field effects. For better visibility, we only show part of this terrace in $2\left(a^{\prime}\right)$ to $2(f)$. Several interesting fs-laser induced changes are observed. First, given the low fluence used (more than 2 orders of magnitude smaller than that used to desorb amorphous solid water from $\operatorname{Pt}(533)$ [1]) the fact that anything at all happens should not be taken for granted. As we have said, at $7 \mathrm{~K}$ the clusters are thermally stable and so the observed changes at such low fluences reveal that the clusters are extremely sensitive to laser radiation. We will show below that at certain fluences most of the clusters are perturbed. Second, some of the smaller clusters diffuse across the surface, while the larger hydrated hexamers are stationary. The motion of the smaller clusters and of single molecules [exemplified in Figs. 3(a) to 3(b)] leads to an increase in
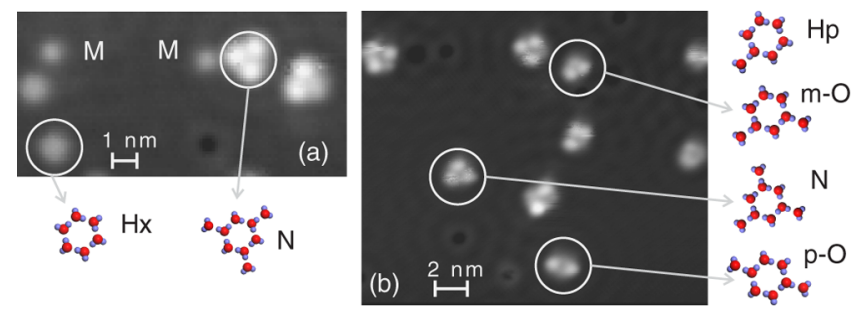

FIG. 1 (color online). STM images and structural models of $\mathrm{D}_{2} \mathrm{O}$ clusters on $\mathrm{Cu}(111)$. Monomer $(\mathrm{M})$, hexamer $(\mathrm{Hx})$, heptamer $(\mathrm{Hp})$, metaoctamer $(\mathrm{m}-\mathrm{O})$, paraoctamer $(\mathrm{p}-\mathrm{O})$, and nonamer $(\mathrm{N})$. The depressions in each STM image are coadsorbed CO molecules. (a) $V=123 \mathrm{mV}, I=16 \mathrm{pA}$; (b) $V=121 \mathrm{mV}$, $I=0.17 \mathrm{nA}$. the number of nonamers during the series of fs illuminations. Third, the clusters remain intact and neither dissociation nor desorption is observed. Finally, the most common event observed upon fs irradiation is a change in structure of the larger hydrated hexamer clusters, specifically the rearrangement of water molecules within the nanoclusters is frequently observed.

We now concentrate on the details of the water molecule rearrangement within the hydrated hexamer clusters. A few specific examples are: The process circled in Figs. 2( $\left(\mathrm{a}^{\prime}\right)$ and $2\left(b^{\prime}\right)$ involves the change in position of the additional molecules of an octamer from para- to metapositions (cf. schematics in Fig. 1). In Fig. 2(b') to 2(c) the additional molecule of a heptamer diffuses on the hexamer from its lower right to its upper left corner. In Fig. 2(d) to 2(f) a process is marked, where a nonamer changes orientation by $60^{\circ}$. In Fig. 3(c) to 3(d) reorientation of a nonamer is shown in higher resolution. Again the nonamer "rotates" by $60^{\circ}$ and, in fact, an apparent $60^{\circ}$ rotation is always observed. The fact that the apparent rotation is always by $60^{\circ}$ actually implies that the entire nanocluster does not rotate, but rather that the peripheral water molecules alone change their binding sites to the underlying (stationary)

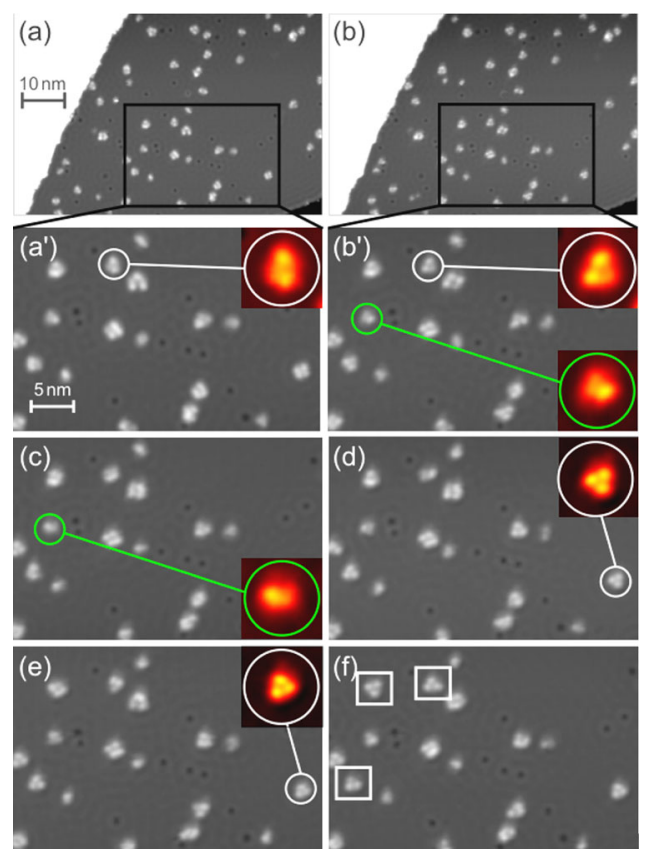

FIG. 2 (color online). STM images $(V=120 \mathrm{mV}, I=17 \mathrm{pA}$ ) out of a series of fs-laser induced changes of $\mathrm{D}_{2} \mathrm{O}$ heptamers, octamers, and nonamers on $\mathrm{Cu}(111)$. For clarity only the part of the terrace marked in (a) and (b) is shown in $\left(a^{\prime}\right)$ to (f). The circled and enlarged clusters in $\left(\mathrm{a}^{\prime}\right)$ to (e) are discussed in the text and the nonamers marked in (f) were octamers in (a). At each step along the series the following parameters $(F, N)$ were applied with $F$ the laser fluence in $\mathrm{J} / \mathrm{m}^{2}$ and $\mathrm{N} \times 10^{8}$ pulses: $(\mathrm{a} \rightarrow \mathrm{b}):(0.55,2) ;(\mathrm{b} \rightarrow \mathrm{c}):(0.44,4) ;(\mathrm{c} \rightarrow \mathrm{d}):(0.39,2) ;(\mathrm{d} \rightarrow \mathrm{e}):$ $(0.18,4)$ and $(0.17,2) ;(\mathrm{e} \rightarrow \mathrm{f}):(0.09,2)$ and $(0.23,6)$. See Ref. [14]. 

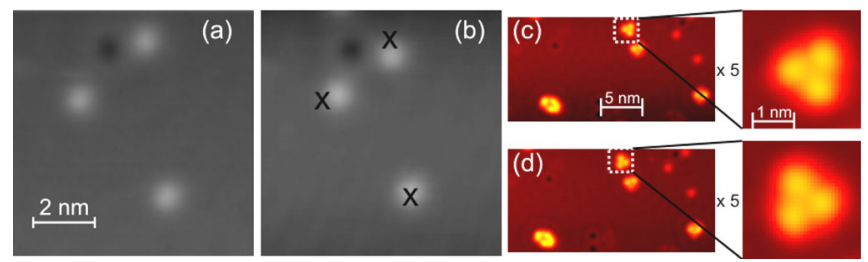

FIG. 3 (color online). STM images of fs-laser induced processes. (a),(b) Three water monomers and a $\mathrm{CO}$ molecule (depression) before and after laser illumination with $0.66 \mathrm{~J} / \mathrm{m}^{2}$ and $1 \times 10^{7}$ pulses $(V=123 \mathrm{mV}, I=16 \mathrm{pA})$; crosses in (b) mark position of the monomers in (a). (c),(d) Rearrangement of a $\mathrm{D}_{2} \mathrm{O}$ nonamer before and after excitation with $0.66 \mathrm{~J} / \mathrm{m}^{2}$ and $2 \times 10^{6}$ pulses $(V=120 \mathrm{mV}, I=16 \mathrm{pA})$.

hexamer. Indeed the change from the ortho to the para structure for the water octamer [Fig. 2(a) to 2(b)] also suggests that it is only the peripheral molecules on top of the hexamer that move upon illumination. To substantiate this suggestion and to get some understanding of the detailed diffusion processes experienced by the water clusters, a series of periodic plane wave DFT calculations were performed with the VASP code [7]. The Perdew, Burke, and Ernzerhof (PBE) [8] exchange-correlation functional was used and transition state structures located with the climbing image nudged elastic band algorithm [9].

Figure 4 shows the computed DFT-PBE energy barriers for (a) rotation of an entire water heptamer and (b) the lowest energy path found for the transfer of the peripheral water molecule of the heptamer from one site of the underlying hexamer to another. The activation energy for the water transfer process is $0.29 \mathrm{eV}$, much lower than the

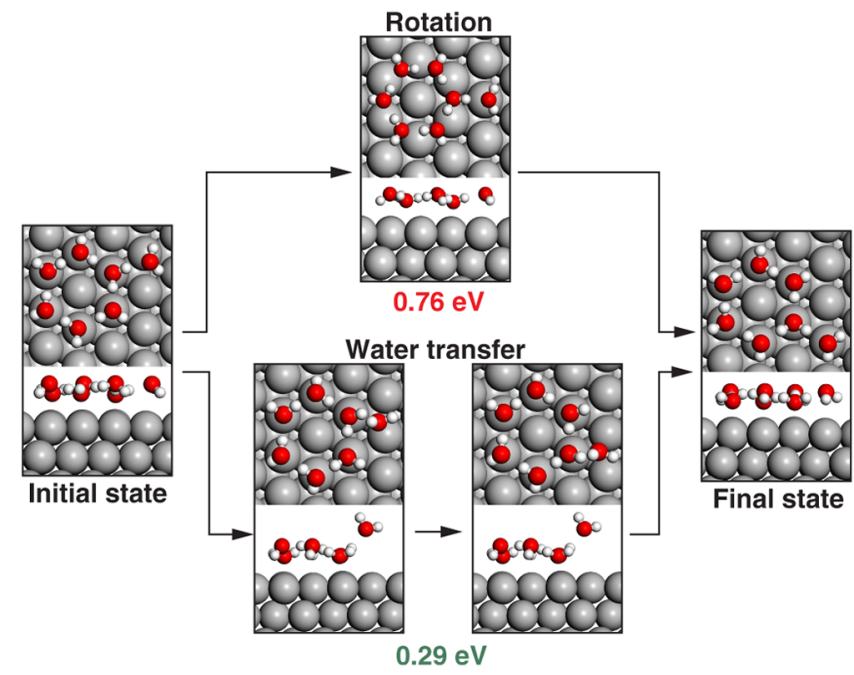

FIG. 4 (color online). DFT determined mechanisms and activation energies for rotation of an entire water heptamer (top path) and transfer of the peripheral water from one site on the underlying hexamer to another (bottom path). The later mechanism is the lowest energy path identified and involves the breaking and reforming of the hydrogen bond between the peripheral water and the underlying hexamer.
$0.76 \mathrm{eV}$ for heptamer rotation. Thus the calculations are consistent with the conclusions drawn from experiment and suggest that upon fs irradiation the peripheral water molecules diffuse across the underlying water hexamers. As can be seen from Fig. 4, the DFT predicted mechanism for the water transfer process is quite interesting and involves rupture of the bond between the peripheral water molecule and the surface, which then "climbs" up and over the water hexamer, breaking and reforming a single hydrogen bond in the process. We caution, however, that since the DFT results reported here are conventional ground-state (adiabatic) calculations they can only be expected to provide general qualitative understanding of plausible experimental processes which, as discussed below, include electronic excitation.

Having explored the details of the fs-induced diffusion of water molecules within the hydrated hexamers, we now quantitatively examine the probability that this process will occur. To this end fs experiments were performed at a range of different fluences $\left(<0.1\right.$ to $\left.>0.5 \mathrm{~J} / \mathrm{m}^{2}\right)$. STM measurements were made of the surface before and after each fs experiment and the rearrangement probability, $P$, determined. A plot of $P$, the proportion of adsorbed clusters which are observed to undergo a rearrangement event, as a function of fluence is given in Fig. 5(a). This reveals that at the highest fluence almost all nanoclusters $(\approx 70 \%)$ have changed. In addition, the dependence of the apparent rearrangement probability on fluence is sublinear. Most likely a sublinear dependence is observed because some rearrangement events go undetected. Specifically, since with STM images are taken only at the beginning and end of each series of femtoscond pulses, multiple rearrangement events that may occur during individual pulses get counted as one event at most. Based on the assumption that there may be multiple unobserved events, it is straightforward via a Poisson distribution, to extract the yield per pulse from the data in Fig. 5(a). Doing this leads to the rearrangement yield per single pulse shown in
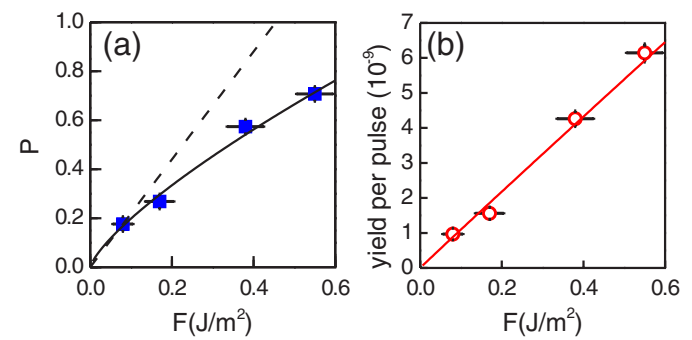

FIG. 5 (color online). Cluster rearrangement as a function of fluence $F$ for $2 \times 10^{8}$ pulses: (a) Proportion of adsorbed clusters, $P$, which are observed in the STM to undergo a rearrangement. The solid line results from a power law fit yielding an exponent of 0.75 ; the dashed line indicates a linear dependence. (b) yield per pulse determined from (a) by considering the Poisson distribution of not observed multiple changes; solid line results from a fit with an exponent of 0.99 . 
Fig. 5(b), which does indeed exhibit a linear dependence in the investigated fluence regime. The latter observation is noteworthy because it suggests that the underlying excitation mechanism is induced by a single electron transition [4]. In such a process an optically excited electron from the metal attaches to the adsorbate, which gains sufficient energy to overcome the relevant reaction barrier directly, here for hydrogen-bond rearrangement. A single electron process such as this is likely to be relevant to atmospheric chemistry, where the intensity of radiation is low, and distinct from the process observed for water desorption from $\operatorname{Pt}(553)$ [1].

Each laser pulse also heats the surface. However, the reaction barrier from DFT $(E=0.29 \mathrm{eV})$ for the lowest energy rearrangement event along with the low fluences employed here make it highly unlikely that the observed cluster rearrangements are thermal events. Specifically, if we cautiously assume that the surface does not resume its original temperature for $t=100 \mathrm{ps}$ of the laser pulse [4], a standard Arrhenius dependence $\left[T=-E /\left(k \cdot \ln \left(Y / t \nu_{0}\right)\right)\right]$ with a typical attempt frequency of $\nu_{0}=10^{12} / \mathrm{s}$ predicts that in order to reach the observed yield of $Y=6 \times 10^{-9}$ at the highest fluence of $0.55 \mathrm{~J} / \mathrm{m}^{2}$ the surface temperature, $T$, must rise to $\approx 140 \mathrm{~K}$. This $\approx 130 \mathrm{~K}$ increase in surface temperature is far above the heating effect expected from femtosecond-laser irradiation at the low fluence employed. For instance a 160 fs-laser pulse of $400 \mathrm{~nm}$ with a fluence of $43 \mathrm{~J} / \mathrm{m}^{2}$ increases the temperature of liquid nitrogen cooled $\mathrm{Cu}(100)$ by only $70 \mathrm{~K}$ [10].

We expect similar diffusion events to occur within the larger ice nanoclusters that form on this surface but have not been discussed here and for water clusters on other surfaces provided the clusters contain molecules that are bound only through one hydrogen bond to the cluster and relatively weakly to the substrate. Examples are the water clusters that have been observed on the (111) surfaces of $\mathrm{Au}$ [11] and Ag [5]. The exact yield will thereby depend on the material properties that determine in turn the hot electrons available for electron attachment. In contrast, for the ice nanostructures that form on $\mathrm{Pd}(111)$ [12] and $\mathrm{Cu}(110)$ [13], which exclusively contain double or triple hydrogen bonded molecules, the energy for the electrons used here is unlikely to be sufficient to induce reorganization of the clusters.

In conclusion, a quantitative study of surface-site specific fs dynamics reveals in connection with density functional theory insight into the hydrogen-bond rearrangement behavior of individual $\mathrm{D}_{2} \mathrm{O}$ molecules within adsorbed water nanoclusters at cryogenic temperatures. Even at the very low fluences examined water diffusion and subsequent clustering, i.e., nonadiabatic coarsening, is induced, revealing that such water clusters are more sensitive to radiation than anticipated. This may be important for the above mentioned ice covered grain particles in Earth's upper atmosphere, where the radiation intensity is low compared to laboratory conditions. The dominant process observed in this study is the diffusion of individual water monomers within the water nanoclusters, a process that involves the breaking and making of hydrogen bonds as well as the bonds with the substrate. From a quantitative analysis of the fluence dependence on the apparent rearrangement probability we suggest that the fs-induced internal hydrogen-bond rearrangements are not thermal, but instead initiated by individual optically excited electrons.

In the broader context this study suggests that in the future it may be possible to select parameters that would allow for the preparation of surfaces with ice nuclei of a selected size, e.g., for reaction studies important in atmospheric chemistry.

K. M.'s work is supported by the DFG, J. C's work by the $\mathrm{AvH}$ foundation and the Royal Society, and A. M's by the EURYI scheme (see www.esf.org/euryi) and the EPSRC. J.C. is grateful to Matthias Scheffler for support, the London Centre for Nanotechnology are warmly acknowledged for the provision of computational resources and Uwe Bovensiepen, FU Berlin, for helpful discussions.

[1] E. H. G. Backus, M. L. Grecea, A. W. Kleyn, and M. Bonn, J. Phys. Chem. B 111, 6141 (2007).

[2] L. Bartels et al., Science 305, 648 (2004).

[3] M. Mehlhorn et al., Rev. Sci. Instrum. 78, 033905 (2007).

[4] X.-L. Zhou, X.-Y. Zhu, and J. M. White, Surf. Sci. Rep. 13, 73 (1991); Ch. Frischkorn and M. Wolf, Chem. Rev. 106, 4207 (2006).

[5] A. Michaelides and K. Morgenstern, Nature Mater. 6, 597 (2007).

[6] K. Morgenstern and K.-H. Rieder, J. Chem. Phys. 116, 5746 (2002).

[7] G. Kresse and J. Hafner, Phys. Rev. B 47, 558 (1993); G. Kresse and J. Furthmüller, Phys. Rev. B 54, 11169 (1996).

[8] J. P. Perdew, K. Burke, and M. Ernzerhof, Phys. Rev. Lett. 77, 3865 (1996); 78, 1396 (1997).

[9] G. Henkelman, B. P. Uberuaga, and H. Jónsson, J. Chem. Phys. 113, 9901 (2000).

[10] L. M. Struck et al., Phys. Rev. Lett. 77, 4576 (1996).

[11] H. Gawronski, K. Morgenstern, and K.-H. Rieder, Eur. Phys. J. D 35, 349 (2005).

[12] J. Cerda et al., Phys. Rev. Lett. 93, 116101 (2004).

[13] J. Carrasco et al., Nature Mater. 8, 427 (2009).

[14] See EPAPS Document No. E-PRLTAO-103-026930 for a series of STM images as shown in Fig. 2 made into a movie. The images are taken at $7 \mathrm{~K}$ with $V=120 \mathrm{mV}$ and $I=17 \mathrm{pA}$. In between the images the STM tip was retracted and a series of $400 \mathrm{~nm}$ fs-laser pulses was given. Number of pulses and fluence are given in the captions of Fig. 2. Note the changes of the peripheral water molecules (most prominent protrusions) of the hydrated hexamers. For more information on EPAPS, see http://www.aip.org/ pubservs/epaps.html. 